PAPER

\title{
Hyperekplexia and stiff-man syndrome: abnormal brainstem reflexes suggest a physiological relationship
}

\author{
S Khasani, K Becker, H-M Meinck
}

J Neurol Neurosurg Psychiatry 2004;75:1265-1269. doi: 10.1136/jnnp.2003.018135

See end of article for authors' affiliations

......................

Correspondence to: Professor Hans-Michael Meinck, Department of Neurology, Im

Neuenheimerfeld 400,

D 69120 Heidelberg,

Germany;

hans-michael_meinck@

med.uni-heidelberg.de

Received 23 May 2003

In revised form

4 December 2003

Accepted5December 2003

\begin{abstract}
Background and objectives: Hyperekplexia and the stiff-man syndrome (SMS) are both conditions with exaggerated startle suggesting abnormal brainstem function. Investigation of brainstem reflexes may provide insight into disturbed reflex excitation and inhibition underlying these movement disorders.

Patients and methods: Using four-channel EMG, we examined four trigeminal brainstem reflexes (monosynaptic masseter, masseter inhibitory, glabella, and orbicularis oculi blink reflexes) and their spread into pericranial muscles in five patients with familial hyperekplexia (FH), two with acquired hyperekplexia $(\mathrm{AH}), 10$ with SMS, and 15 healthy control subjects.

Results: Both FH/AH and SMS patients had abnormal propagation of brainstem reflexes into pericranial muscles. All patients with hyperekplexia showed an abnormal short-latency $(15-20 \mathrm{~ms})$ reflex in the trapezius muscle with a characteristic clinical appearance ("head retraction jerk") evoked by tactile or electrical stimulation of the trigeminal nerve, but normal monosynaptic masseter reflexes. Inhibitory brainstem reflexes were attenuated in some $\mathrm{FH} / \mathrm{AH}$ patients. Four of 10 patients with SMS had similar short-latency reflexes in the neck muscles and frequently showed widespread enhancement of other excitatory reflexes, reflex spasms, and attenuation of inhibitory brainstem reflexes.

Conclusion: Reflex excitation is exaggerated and inhibition is attenuated in both stiff-man syndrome and familial or acquired hyperekplexia, indicating a physiological relationship. Reflex transmission in the brainstem appears biased towards excitation which may imply dysfunction of inhibitory glycinergic or GABAergic interneurons, or both.
\end{abstract}

speculative. ${ }^{16}$ We therefore electromyographically investigated reflex excitation and inhibition at the brainstem level. We examined four brainstem reflexes, all of which are well established in clinical or electrodiagnostic testing: the monosynaptic masseter reflex, the masseter inhibitory reflex, the glabella reflex, and the blink reflex.

\section{METHODS}

\section{Patients and controls}

We investigated five patients with $\mathrm{FH}$ (2-36 years of age) from three families, two patients with $\mathrm{AH}$ ( 16 and 33 years of age), 10 patients with SMS (31-73 years of age), and 15 healthy control subjects (CS; 21-53 years of age). The subjects or, where legally required, their parents gave informed consent to participate in electrophysiological studies. All FH patients participating in this study carried point mutations of the GLRAl gene. The two patients with $\mathrm{AH}$ had no neurological abnormality other than hyperekplexia, gait disturbance, and space phobia, as well as normal ancillary findings including brain MRI, CSF analysis, and screening for GAD-Ab. Four patients with $\mathrm{FH}$ and both $\mathrm{AH}$ patients were on symptomatic treatment with clonazepam (0.75-6 mg per day) or diazepam (6 mg per day). All SMS patients had stiffness of the legs and trunk; one patient had additional involvement of the neck and arms. In none of them did stiffness or spasms involve the face. In eight of the 10 SMS patients, radioimmunoassay testing disclosed serum antibodies directed against GAD. Nine patients with SMS were under current symptomatic treatment with diazepam (5-60 mg per day), clonazepam (7 mg per day), intrathecal

Abbreviations: $\mathrm{AH}$, acquired hyperekplexia; $\mathrm{CS}$, control subject; $\mathrm{FH}$, familial hyperekplexia; MAS, masseter; OOC, orbicularis oculi; SCM, sternocleidomastoid; SMS, stiff-man syndrome; TRA, trapezius glycinergic inhibition. However, the physiologic mechan isms underlying similar phenomena in AH and SMS are 
Table 1 Survey of abnormal reflex excitation and inhibition in patients with familial or acquired hyperekplexia or with stiff-man syndrome

\begin{tabular}{|c|c|c|c|}
\hline Stimulus & & $\begin{array}{l}\mathrm{FH} / \mathrm{AH} \\
(\mathrm{n}=7)\end{array}$ & $\begin{array}{l}\text { SMS } \\
(n=10)\end{array}$ \\
\hline Tap to chin & $\begin{array}{l}\text { Abnormal reflex excitation } \\
\text { Monosynaptic MAS reflex } \\
\text { exaggerated }\end{array}$ & $0 / 7$ & $2 / 10$ \\
\hline & Short-latency spread into TRA/SCM & $7 / 7$ & $2 / 10$ \\
\hline Pulse to V3 & Short-latency spread into TRA/SCM & 7/7 & $0 / 10$ \\
\hline Tap to glabella & Short-latency spread into TRA/SCM & $7 / 7$ & $4 / 10$ \\
\hline & Widespread reflex spasms & $0 / 7$ & $5 / 10$ \\
\hline Pulse to V1 & Short-latency spread into TRA/SCM & $7 / 7$ & $0 / 10$ \\
\hline Any & Widespread reflex spasms & $0 / 7$ & $5 / 10$ \\
\hline Tap to chin & $\begin{array}{l}\text { Abnormal reflex inhibition } \\
\text { Loss of reflex inhibition in TRA/SCM } \\
\text { MAS post reflex silent period } \\
\text { incomplete/delayed }\end{array}$ & $\begin{array}{l}1 / 7 \\
2 / 7\end{array}$ & $\begin{array}{l}3 / 10 \\
3 / 10\end{array}$ \\
\hline Pulse to V3 & $\begin{array}{l}\text { Loss or delay of S2 component of } \\
\text { MAS inhibition }\end{array}$ & $2 / 7$ & $1 / 10$ \\
\hline & $\begin{array}{l}\text { Elevated reflex thresholds for MAS } \\
\text { inhibition }\end{array}$ & $0 / 7$ & $7 / 10$ \\
\hline Tap to glabella & Loss of $\mathrm{OOC}$ inhibition & $0 / 7$ & $2 / 10$ \\
\hline
\end{tabular}

MAS, masseter; OOC, orbicularis oculi; SCM, sternocleidomastoideus muscles; TRA, trapezius; V1/V3, first/third trigeminal nerve branch.

baclofen (60-925 $\mu$ g per day), or tizanidine (16 mg per day), or a combination of these drugs.

\section{Procedure}

Subjects rested on a comfortable couch in supine position with their eyes closed. The glabella reflex was elicited by gently tapping the root of the nose with a micro switchequipped tendon hammer. The masseter reflex and the masseter post reflex silent period were evoked by tapping the tip of the chin. The blink reflex was elicited by electrical stimulation of the left supraorbital nerve (square wave shocks, pulse width $0.2 \mathrm{~ms}$ ). The masseter inhibitory reflex was evoked by electrical stimulation of the lower lip. In the case of electrical stimulation, we first determined the individual reflex thresholds by a stepwise current increase or decrease. We defined the threshold as the lowest required stimulus intensity to consistently reproduce at least one reflex component. We then elicited the orbicularis oculi blink and the masseter inhibitory reflexes with a stimulus intensity of 2-3 $\times$ reflex threshold. Because of altered reflex thresholds, considerably higher stimulus intensities were required in some SMS and $\mathrm{FH} / \mathrm{AH}$ patients.

In addition to the reflex effects in the respective target muscle, we investigated the spread of reflexes into neighbouring muscles. Therefore, we recorded brainstem reflexes simultaneously from the orbicularis oculi (OOC), masseter (MAS), trapezius (TRA), and sternocleidomastoid (SCM) muscles on the left side using surface electrodes (diameter $7 \mathrm{~mm}$ ) attached over the respective muscles (filter setting 100-3000 Hz). Four reflexes were elicited consecutively at intervals exceeding $30 \mathrm{~s}$ and electronically superimposed.

Normal values were obtained from the 15 CS subjects (fig 1). Quantitative data are presented as absolute values or arithmetic means with 1 SD. The level of significance was set at $\mathrm{p}<0.001$.

\section{RESULTS \\ Familial hyperekplexia}

\section{Reflex excitation}

The blink, glabella, and monosynaptic masseter reflexes were normal. However, all patients had abnormal irradiation of excitatory reflexes into the neck muscles: both electrical and mechanical stimulation elicited highly synchronised shortlatency reflex activity in the TRA muscle (table 1, fig $2 \mathrm{C}$ and D). The reflex latency was shorter with taps to the jaw (12.6$16.0 \mathrm{~ms})$ than to the glabella (13.7-22.1 ms), and the amplitudes higher and more stable with mechanical than with electrical stimulation. A second, less synchronised component followed inconstantly at around $50 \mathrm{~ms}$. In all FH patients, a synchronised but unstable reflex in the SCM muscle accompanied the TRA reflex with slightly longer latencies after taps to the chin (16.2-25.5 ms) and to the glabella (17.6-25.2 ms). Such activation of the neck muscles was only sporadically observed in the CS: upon elicitation of the blink reflex, two CS had early trapezius reflexes at a similar latency (20 ms; fig ID) but with smaller amplitudes, and four CS exhibited late responses (latency around $60 \mathrm{~ms}$ ).

\section{Reflex inhibition}

Besides these excitatory reflex abnormalities, pre-activation of the pericranial muscles revealed lost or diminished inhibitory reflexes in the $\mathrm{FH}$ subjects: the exteroceptive suppression of the OOC, intercalated between the R1 and R2 components of the blink reflex ${ }^{17}{ }^{18}$ in all CS (latency (SD) 21.7 (1.5) $\mathrm{ms})$, was delayed in one patient $(30.0 \mathrm{~ms}, \mathrm{p}<0.001)$. Similarly, the masseter post reflex silent period was

\section{A Masseter reflex}

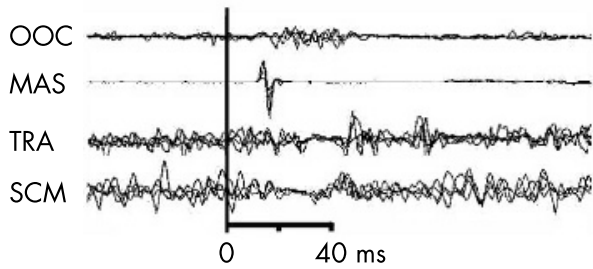

C Masseter inhibitory reflex

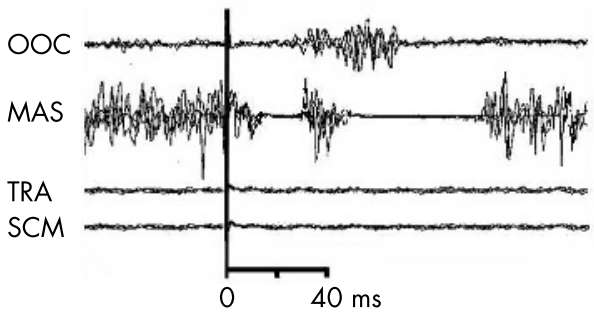

B Glabella reflex

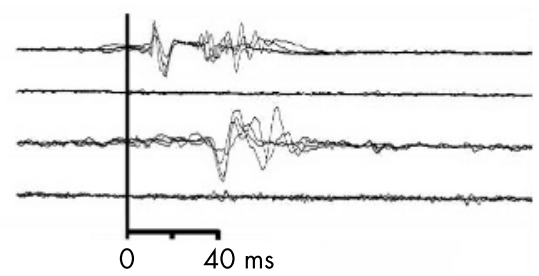

D Blink reflex

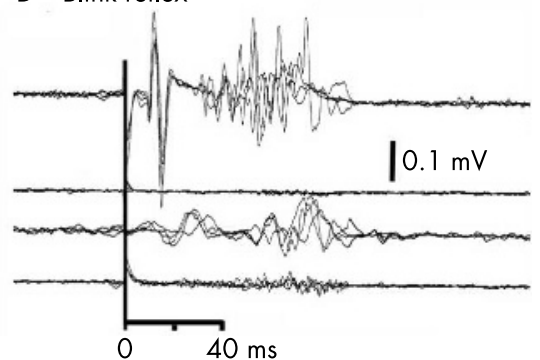

Figure 1 Normal patterns of brainstem reflexes recorded simultaneously from the orbicularis oculi (OOC), masseter (MAS), trapezius (TRA), and sternocleidomastoideus (SCM) muscles. In each panel, four reflex responses are superimposed. In A, voluntary pre-innervation of the TRA and SCM during elicitation of the masseter reflex allows identification of the inhibitory reflex components in these muscles. Reflex activation of the TRA in the glabella or the blink reflex, though uncommon in normal subjects (table 1), is shown here for comparison with the abnormal reflex patterns in patients with $\mathrm{FH} / \mathrm{AH}$ (fig 2) and SMS (fig 3). The vertical calibration (bottom right) applies to all registrations. 
Glabella reflex

Acquired hyperekplexia

A

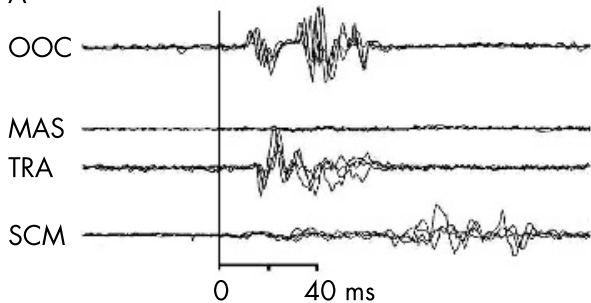

Blink reflex

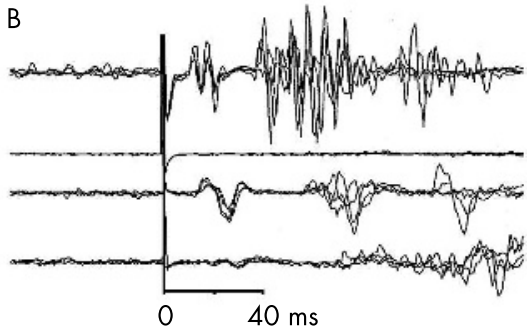

Figure 2 Abnormal spread of the glabella and blink reflexes in a patient with acquired hyperekplexia (A, B) and in a patient with familial hyperekplexia (C, D). Abbreviations as in fig 1. In contrast to the control subjects, shortlatency reflex activation of the TRA is regularly observed in both conditions. The vertical calibration in $\mathrm{C}$ applies to all registrations.
C

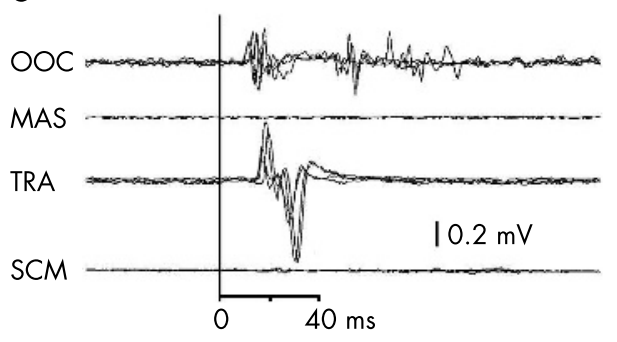

Familial hyperekplexia

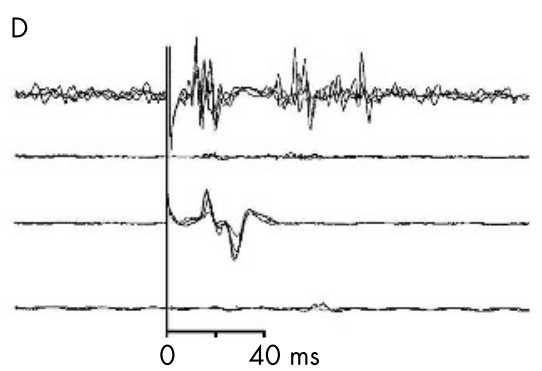

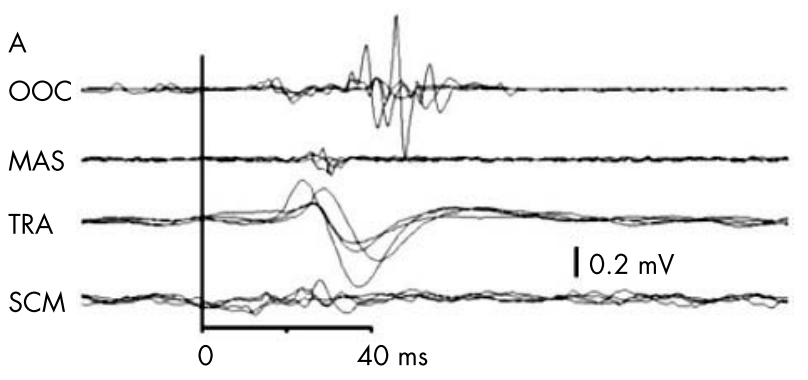
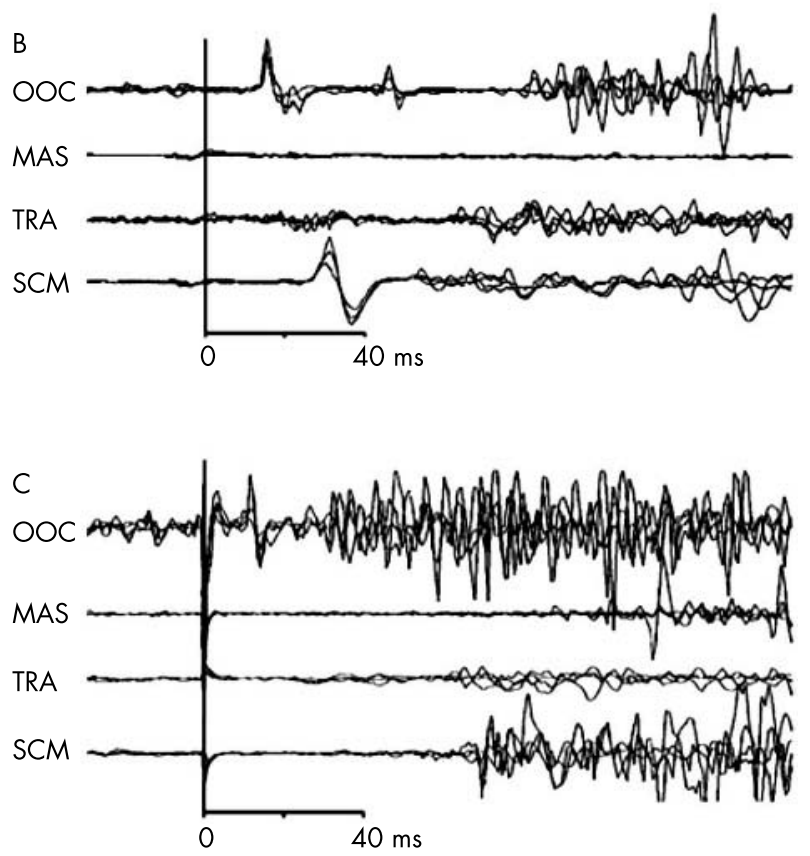

Figure 3 Abnormal glabella $(A, B)$ and blink $(C)$ reflex patterns in three patients with SMS: short-latency responses of the TRA (A, B) and SCM (B), abnormal synchronisation of the second OOC reflex component (B), and reflex spasms in the OOC and SCM (C). Abbreviations as in fig 1. The vertical calibration in A applies to all registrations. incomplete in one patient. In the same patient, the S2 component of the electrically evoked masseter inhibitory reflex could not be elicited, even at maximum tolerable stimulus intensity $(62 \mathrm{~mA})$. Moreover, reflex inhibition of the TRA muscle, observed in all CS after a chin tap (latency 30.3 (6.1) ms; fig 1A), was overrun by the hypersynchronous excitatory TRA reflex in FH and followed by a long-lasting (38-4l ms) suppression of the ongoing EMG activity.

\section{Acquired hyperekplexia}

The reflex irradiation into the TRA and SCM muscles had a similar appearance and latency as in patients with FH (fig 2A and B). The exteroceptive suppression of the OOC was lost and S2 of the masseter inhibitory reflex was delayed to $68.0 \mathrm{~ms}(\mathrm{p}<0.001)$ in one patient with $\mathrm{AH}$.

\section{Stiff-man syndrome}

\section{Reflex excitation}

The monosynaptic masseter reflex was exaggerated in one patient with SMS (amplitude $2.19 \mathrm{mV}$, mean (SD) for CS: 0.37 (0.39); $\mathrm{z}>4$ ). In another, the masseter reflex could be reproducibly elicited with taps to the glabella, compatible with enhanced gain of reflex transmission. ${ }^{19}$

In four patients, taps to the glabella elicited hypersynchronous reflexes with short latencies (24.8-28.5 ms) and high amplitudes (fig 3A and B) in the TRA and SCM muscles, resembling the abnormal reflexes in $\mathrm{FH} / \mathrm{AH}$. In five patients, both mechanical and particularly electrical stimulation of the face evoked distinct late reflex effects in the TRA and SCM muscles with latencies varying between 35 and $71 \mathrm{~ms}$ and with widespread desynchronised and long-lasting muscle activity (fig 3C) considered reflex spasms. ${ }^{19}$ The presence of these observed reflex alterations, in particular the early reflex activation of the TRA and SCM muscles, was not associated with the absence or presence of GAD-Ab.

\section{Reflex inhibition}

In three patients the latency of the masseter post reflex silent period was delayed beyond $20 \mathrm{~ms}$ (mean (SD) for CS: 12.4 (2.3) ms; $\mathrm{p}<0.001 ; \mathrm{z}>3$ ). The electrically evoked masseter inhibition had normal latency and duration, but required abnormally high stimulus intensities: in five patients, the 
reflex threshold was greater than $41 \mathrm{~mA}$ (compared to 19.4 (4.4) $\mathrm{mA}$ in $\mathrm{CS} ; \mathrm{p}<0.001$ ). Taps to the chin failed to induce reflex inhibition of the TRA in two patients, and of the SCM (latency in CS, 21.0 (4.5) ms) in one SMS patient. Both reflex effects were regularly observed in CS (fig 1A). Similarly, the exteroceptive inhibition of the OOC muscle was abolished in two patients with SMS.

\section{DISCUSSION}

In patients with $\mathrm{FH} / \mathrm{AH}$ and SMS, the patterns of normal brainstem reflexes with their irradiation into pericranial muscles are distinctively altered. In both motor disturbances, we found enhanced reflex excitation and attenuated reflex inhibition. Exclusively in patients with SMS, moreover, we observed hyperactive masseter reflexes and widespread reflex spasms. While some of the presented abnormalities of excitatory reflexes have been documented in other reports, ${ }^{16}{ }^{20-22}$ this is the first study to systematically evaluate inhibitory brainstem reflexes in patients with $\mathrm{FH} / \mathrm{AH}$ and SMS. Our findings show that in both disorders reflex transmission through the brainstem is biased towards excitation.

Our study has certain limitations. First, most examined patients were on treatment with benzodiazepines or baclofen which could have altered reflex transmission and possibly given false EMG findings. However, as these drugs are known to attenuate reflex excitation and enforce inhibition, ${ }^{23-25}$ one might expect a higher prevalence of reflex abnormalities in untreated patients rather than falsely positive findings. Second, no normative data for brainstem reflexes exist in children. However, personal experience and anecdotal reports ${ }^{9}$ suggest that the patterns and latencies principally resemble those in adults. Third, the low prevalence of both syndromes and the small number of examined patients limit the ability to generalise our findings.

\section{Abnormal brainstem reflexes in familial hyperekplexia}

In our study, the most prominent reflex alteration was the hypersynchronous short-latency reflex in the TRA muscle. Because of its characteristic clinical appearance, we will refer to it as the "head retraction reflex". ${ }^{16}{ }^{26}$ The fact that it can be elicited by electrical stimulation of the trigeminal nerve suggests that this trigemino-cervical reflex belongs to the cutaneo-muscular reflexes. ${ }^{27}$ Its latency and pattern resemble the trigemino-facial blink reflex. By analogy, we assume that similar neuronal pathways link the trigeminal sensory nuclei with the spinal motor nuclei of the accessory nerve. ${ }^{28}{ }^{29}$ The presence of a trace of this reflex in some CS (fig lD) ${ }^{30}$ andas revealed by needle electrode recordings-also in the deep neck muscles of normal subjects ${ }^{31}$ suggests that the head retraction reflex may be rudimentarily present, but physiologically suppressed in normal subjects. Since this reflex is the most prominent, and often the only firm neurological sign of $\mathrm{FH}_{1}{ }^{12}$ a disorder of the inhibitory glycine receptor, ${ }^{79}$ it is tempting to speculate that disinhibition of this reflex in FH is due to defective glycinergic inhibition. The presence of a clinically and electrophysiologically similar head retraction reflex in patients with $\mathrm{AH}$ suggests that $\mathrm{FH}$ and $\mathrm{AH}$ share physiological abnormalities.

\section{Abnormal brainstem reflexes in the stiff-man syndrome}

The head retraction reflex was also present in four of 10 patients with SMS corresponding to a prevalence of about $50 \%$ in a recent large cohort study. ${ }^{16}$ Another characteristic finding observed only in our patients with SMS, but described in patients with tetanus or in hemimasticatory spasm, ${ }^{32}{ }^{33}$ were widespread reflex spasms. None of the SMS patients investigated had clinical involvement of the face, and the neck was involved in only one. Therefore the high prevalence of abnormal trigeminal reflexes suggests subclinical involvement of the brainstem. Excitability of the reflex circuits between the trigeminal sensory nuclei and the motor nuclei of the cranial nerves is modulated by segmental and suprasegmental inputs. ${ }^{34}{ }^{35}$ Increased brainstem interneuronal excitability in SMS patients as revealed by enhanced blink reflex recovery cycles was ascribed to altered inhibitory drive from the basal ganglia or to cortical hyperexcitability. ${ }^{12}{ }^{36}$ Intrinsic pathology of brainstem interneurons was considered unlikely, because the masseteric exteroceptive silent period in patients with SMS has been reported as normal. ${ }^{32}{ }^{36}$ Our findings differ from the reported data in that a considerable proportion of patients with SMS do have abnormal masseteric inhibitory components suggesting primary dysfunction of the inhibitory interneurons at brainstem level.

\section{A physiological relationship?}

Reflex transmission through the brainstem is biased towards excitation in both SMS and FH. Both motor disturbances also have in common the presence of a head retraction reflex. ${ }^{16}$ Such similarities suggest a physiological relationship. Are these similarities a reflection of a shared synaptic disease process between SMS and FH?

In FH, a genetic disorder with mutated glycine receptors, abnormalities of brainstem reflexes are most likely due to diminished glycine inhibition. ${ }^{37}$ SMS, however, is currently understood as a disorder with impaired GABAergic synaptic transmission. ${ }^{11} 38$

Biochemical and physiological links between SMS and FH not yet understood are that cerebrospinal fluid levels of free GABA are reduced in $\mathrm{FH}^{39}$ and segmental glycinergic inhibitory circuits are impaired in SMS. ${ }^{13}$ A simple explanation for such a relationship might be that the inhibitory transmitters, GABA and glycine are simultaneously present in the trigeminal subnuclei $i^{40-43}$ and are both involved in the trigeminal reflex transmission. ${ }^{3}$ This hypothesis is corroborated by the observation that in various species, and presumably also in human beings, $\mathrm{GABA}_{\mathrm{A}}$ and glycine receptors show a widespread co-localisation in the central nervous system. ${ }^{44-47}$

\section{CONCLUSIONS}

Patients with the familial or acquired forms of hyperekplexia and patients with the stiff-man syndrome share physiological abnormalities: brainstem reflex excitation is exaggerated whereas reflex inhibition is attenuated. This is likely due to defective inhibition, mediated by GABAergic and glycinergic brainstem interneurons. We conclude that both transmitters are co-localised in polysynaptic reflex pathways of the brainstem.

\section{ACKNOWLEDGEMENTS}

The authors are indebted to Professor C-M Becker, Erlangen, Germany, for helpful discussion and for providing molecular genetic analysis in patients with familial hyperekplexia and to K Dinkel, PhD, Heidelberg, Germany, for providing radioimmunoassay testing for GAD autoantibodies in patients with stiff-man syndrome and acquired hyperekplexia.

\section{Authors' affiliations}

S Khasani, H-M Meinck, Department of Neurology, University of Heidelberg, Germany

S Khasani, Department of Neurology, Tulane University, New Orleans, LA, USA

K Becker, Department of Biochemistry and Molecular Medicine, University of Erlangen, Germany 
Volkswagenstiftung, Hannover, Germany, provided generous financial support.

Competing interests: none declared

\section{REFERENCES}

1 Suhren O, Bruyn G, Tuynman J. In: Hyperekplexia. A hereditary startle syndrome. J Neurol Sci 1966;3:577-605.

2 Tijssen M. Hyperekplexia-startle disease. In: Neurology. Leiden: Riiksuniversiteit Leiden, 1997: 1-173.

3 Barker R, Revesz T, Thom M, et al. Review of 23 patients affected by the stiff man syndrome: clinical subdivision into stiff trunk (man) syndrome, stiff limb syndrome, and progressive encephalomyelitis with rigidity. J Neurol Neurosurg Psychiatry 1998;65:633-40.

4 Matsumoto J, Hallett M. Startle syndromes. In: Marsden CD, Fahn S, eds. Movement disorders 3. London: Butterworth Heinemann, 1996:418-33.

5 Henningsen $\mathrm{P}$, Meinck H-M. Specific phobia is a frequent non-motor feature in stiff man syndrome. J Neurol Neurosurg Psychiatry 2003;74:462-5.

6 Meinck HM, Thompson PD. Stiff man syndrome and related conditions. Mov Disord 2002; 17:853-66.

7 Shiang R, Ryan S, Zhu Y. Mutations in the alpha 1 subunit of the inhibitory glycine receptor cause the dominant neurologic disorder, hyperekplexia. Nat Genet 1993;5:351-8.

8 Shiang R, Ryan S, Zhu YZ, et al. Mutational analysis of familial and sporadic hyperekplexia. Ann Neurol 1995;38:85-91.

9 Brune W, Weber R, Saul B, et al. A GLRA1 null mutation in recessive hyperekplexia challenges the functional role of glycine receptors. Am J Hum Genet 1996;58:989-97.

10 Brown P, Rothwell J, Thompson P, et al. The hyperekplexias and their relationship to the normal startle reflex. Brain 1991:114:1903-28.

11 Levy L, Dalakas $M$, Floeter $M$. The stiff-person syndrome: an autoimmune disorder affecting neurotransmission of gamma-aminobutyric acid. Ann Intern Med 1999;131:522-30

12 Meinck H, Ricker K, Conrad B. The stiff man syndrome: new pathophysiological aspects from abnormal exteroceptive reflexes and the response to clomipramine, clonidine, and tizanidine. J Neurol Neurosurg Psychiatry 1984;47:280-7.

13 Meinck $H$, Ricker $K$, Hülser $P$, et al. Stiff man syndrome: neurophysiological findings in eight patients. J Neurol 1995;242:134-42.

14 Floeter M, Valls-Sole J, Toro C, et al. Physiologic studies of spinal inhibitory circuits in patients with stiff-person syndrome. Neurology 1998;51:85-93.

15 Sandbrink F, Syed N, Fujii M, et al. Motor cortex excitability in stiff-person syndrome. Brain 2000;123:2231-9.

16 Berger C, Meinck H-M. Head retraction reflex in stiff-man syndrome and related disorders. Mov Disord 2003;18:906-11.

17 Leon F, Arimure K, Osame M. Three silent periods in the orbicularis oculi muscles of man: normal findings and some clinical vignettes. Electromyogr Clin Neurophysiol 2001;41:393-400.

18 Berardelli A, Cruccu G, Kimura J, et al. The orbicularis oculi reflexes. The International Federation of Clinical Neurophysiology. Electroencephalogr Clin Neurophysiol Suppl 1999;52:249-53.

19 Lance J, De Gail P. Spread of phasic muscle reflexes in normal and spastic subjects. J Neurol Neurosurg Psychiatry 1965;28:328-34.

20 Alberca R, Romero M, Chaparro J. Jerking stiff-man syndrome. J Neurol Neurosurg Psychiatry 1982;45:1159-60.

21 Leigh P, Rothwell J, Traub M, et al. A patient with reflex myoclonus and muscle rigidity: "jerking stiff-man syndrome". I Neurol Neurosurg Psychiatry 1980;43:1125-31.

22 Matsumoto J, Fuhr P, Nigro M, et al. Physiological abnormalities in hereditary hyperekplexia. Ann Neurol 1992;32:41-50.

23 Leardi MG, Ferracuti $S$, Innocenti $P$, et al. Modulation using racemic and levorotary baclofen of the trigeminal reflex in man. Boll Soc ltal Biol Sper $1990 ; 66: 272-7$.
24 Seitz RJ, Blank B, Kiwit JCW, et al. Stiff-person syndrome with anti-glutamic acid decarboxylase autoantibodies: complete remission of symptoms after intrathecal baclofen administration. J Neurol 1995;242:618-22.

25 Cruccu G, Ferracuti S, Leardi MG, et al. Nociceptive quality of the orbicularis oculi reflexes as evaluated by distinct opiate- and benzodiazepine-induced changes in man. Brain Res 1991;556:209-17.

26 Wartenberg R. Head retraction reflex. Am J Med Sci 1941;201:553-61.

27 Duensing F. Zur Pathologie der exteroceptiven Reflexe des Menschen. J Nerv Ment Dis 1952; 116:973-87.

28 Rossi B, Pasca S, Sartucci F, et al. Trigemino-cervical reflex in pathology of the brain stem and of the first cervical cord segments. Electromyogr Clin Neurophysiol 1989;29:67-71

29 Matsushita M, Ikeda M, Okado N. The cells of origin of the trigeminothalamic, trigeminospinal and trigeminocerebellar projections in the cat. Neuroscience 1982;7:1439-54

30 Nakashima K, Thompson P, Rothwell J, et al. An exteroceptive reflex in the sternocleidomastoid muscle produced by electrical stimulation of the supraorbital nerve in normal subjects and patients with spasmodic torticollis. Neurology 1989;39:1354-8

31 Ertekin C, Celebisoy N, Uludag B. Trigemino-cervical reflexes in normal subjects. J Neurol Sci 1996;143:84-90.

32 Auger RG. AAEM minimonograph \#44: diseases associated with excess motor unit activity. Muscle Nerve 1994;17:1250-63.

33 Cruccu G, Inghilleri M, Berardelli A, et al. Pathophysiology of hemimasticatory spasm. J Neurol Neurosurg Psychiatry 1994;57:43-50.

34 Holstege G, Kuypers H, Dekker J. The organization of the bulbar fibre connections to the trigeminal, facial and hypoglossal motor nuclei. II. An autoradiographic tracing study in cat. Brain 1977;100:264-86.

35 Kimura J. The blink reflex. In: Electrodiagnosis in diseases of nerve and muscle: principles and practice. New York: Oxford University Press, 2001:409-38.

36 Molloy FM, Dalakas MC, Floeter MK. Increased brainstem excitability in stiffperson syndrome. Neurology 2002;59:449-51.

37 Saul B, Kuner T, Sobetzko D, et al. Novel GLRA1 missense mutation (P250T) in dominant hyperekplexia defines an intracellular determinant of glycine receptor channel gating. J Neurosci 1999;19:869-77.

38 Dinkel K, Meinck H, Jury K, et al. Inhibition of gamma aminobutyric acid synthesis by glutamic acid decarboxylase autoantibodies in stiff man syndrome. Ann Neurol 1998;44:194-201.

39 Dubowitz L, Bouza H, Hird M, et al. Low cerebrospinal fluid concentration of free gamma-aminobutyric acid in startle disease. Lancet 1992;340:80-1

40 Ginestal E, Matute C. Gamma-aminobutyric acid-immunoreactive neurons in the rat trigeminal nuclei. Histochemistry 1993;99:49-55.

41 Turman J, Chandler S. Immunohistochemical evidence for GABA and glycinecontaining trigeminal premotoneurons in the guinea pig. Synapse 1994; 18:7-20.

42 Dumba J, Irish PS, Anderson NL, et al. Electron microscopic analysis of gamma-aminobutyric acid and glycine colocalization in rat trigeminal subnucleus caudalis. Brain Res 1998;806:16-25

43 Takahashi O, Satoda T, Uchida T. Distribution of GABA-immunoreactive premotor neurons projecting to the trigeminal motor nucleus in the rat. $J$ Hirnforsch 1995;36:203-8.

44 Boucher Y, Pollin B, Azerad J. Microinfusions of excitatory amino acid antagonists into the trigeminal sensory complex antagonize the jaw opening reflex in freely moving rats. Brain Res 1993:614:155-63.

45 Bohlhalter $\mathrm{S}$, Mohler $\mathrm{H}$, Fritschy J-M. Inhibitory neurotransmission in rat spinal cord: co-localization of glycine- and GABA-A-receptors at GABAergic synaptic contacts demonstrated by triple immunofluorescence staining. Brain Res 1994:642:59-69.

46 Maxwell D. Colocalization of glycine and GABA in synapses on spinomedullary neurons. Brain Res 1995;690:127-32.

47 Jonas P, Bischofberger J, Sandkühler J. Corelease of two fast neurotransmitters at a central synapse. Science 1998;281:419-24. 